\title{
Article
}

\section{Design and Construction of NACA-4415 Airfoil with Various Shaped Surface Modifications}

\author{
Rubiat Mustak \\ Department of Mechanical Engineering, Khulna University of Engineering \& Technology, Khulna-9203, \\ Bangladesh; rubiat_kuet@me.kuet.ac.bd
}

\begin{abstract}
A fluid flowing over an object has a tendency to drag the object along it's flow direction. An object passing through a fluid which is stationary there is a tendency to slow the object down. For a stationary object in a fluid which is flowing there is a tendency to move the object in the fluid flowing direction. These tendencies of flowing fluid is known as drag. While moving through air airplanes also subjected to several drags. Airplanes subjected to pressure drag or form drag due to flow separation which is based on the pressure difference between the upstream and downstream surfaces of the object. Airplanes also subjected to Skin friction drag which results from the viscous shear of the fluid flowing over the object surface. In order to overcome these drags airplane wings cross section airfoils are designed very carefully. National Advisory Committee for Aeronautics, or NACA, developed and tested "families" of airfoils. Some of the most successful of these were the NACA four-digit and five-digit series. The necessary coordinates for designing NACA airfoil profiles are available in online. UIUC also provide coordinates for designing NACA airfoil profiles. But the present work describes the way of designing NACA four digit airfoils without taking any coordinates from available sources like google or any other search engines. Using $C$ programming with the help of NACA provided equations a generalized source code is designed . Which will provide coordinates for designing any NACA four digit airfoil profiles.With the help of this obtained profile the wing model is also constructed using solid works. Using solid works model the real model was constructed using wood. The chord of regular surface airfoil is $21 \mathrm{~cm}$ and the span is also $21 \mathrm{~cm}$. The airfoil profile taken for the model construction is NACA- 4415 which is a four digit cambered airfoil.The present work also show some figures of an airfoil by applying certain surface modifications in form of dimples.
\end{abstract}

Keywords: airfoil; dimple; drag; lift.

\section{Introduction}

An airplane is a vehicle with wings and one or more engines that enable it to fly through the air and any section of the wing cut by the plane which is perpendicular to the wing is called an airfoil. Airplane wings produce lift by creating pressure difference between top surface and bottom surface. For an object passing through a fluid which is stationary there is a tendency to slow the object down. While moving through air airplanes also subjected to several drags. In order to overcome these drags airplane wings cross section airfoils are designed and studied for many years. Ludwig Prandtl in the year of 1917 made a wind tunnel at Göttingen which is capable of testing large airfoil sections. He also had a model which is based on mathematics to generate airfoil like curves. U.S. National Advisory Committee for Aeronautics, or NACA, in the year of 1930 prepared and investigate"families" of airfoils. Some of the very popular of these were the NACA four-digit and five-digit series. NACA's six digit airfoil 23000 series which was prepared in 1935, is assumed to be the most widely used airfoil in history. Nearly 1940, development of airfoils had completed several milestones. The first was not due to any single investigator, that camber helps the production of lift. The second one was the invention, by Prandtl that thickness more than a tenth of the chord length was good for producing lift.The last one was by Eastman Jacobs who classified the airfoils into 
"families" which allowed producers to choose airfoil profiles from a catalog. The most recent development was a the relationship between mathematics and designing of airfoils. Now airfoils specifically designed for flight near the sound velocity. These are supercritical airfoils which have special design features to withstand shock waves.

\section{Airfoil Design}

The airfoil profiles of all NACA families obtained by merging a mean line and a thickness distribution. The needed coordinates for the distribution of mean lines and thickness is obtained from the available sources. The available sources provides with necessary coordinates. The way of merging a mean line and a thickness distribution to produce the required airfoil profile is shown by below mentioned figure.

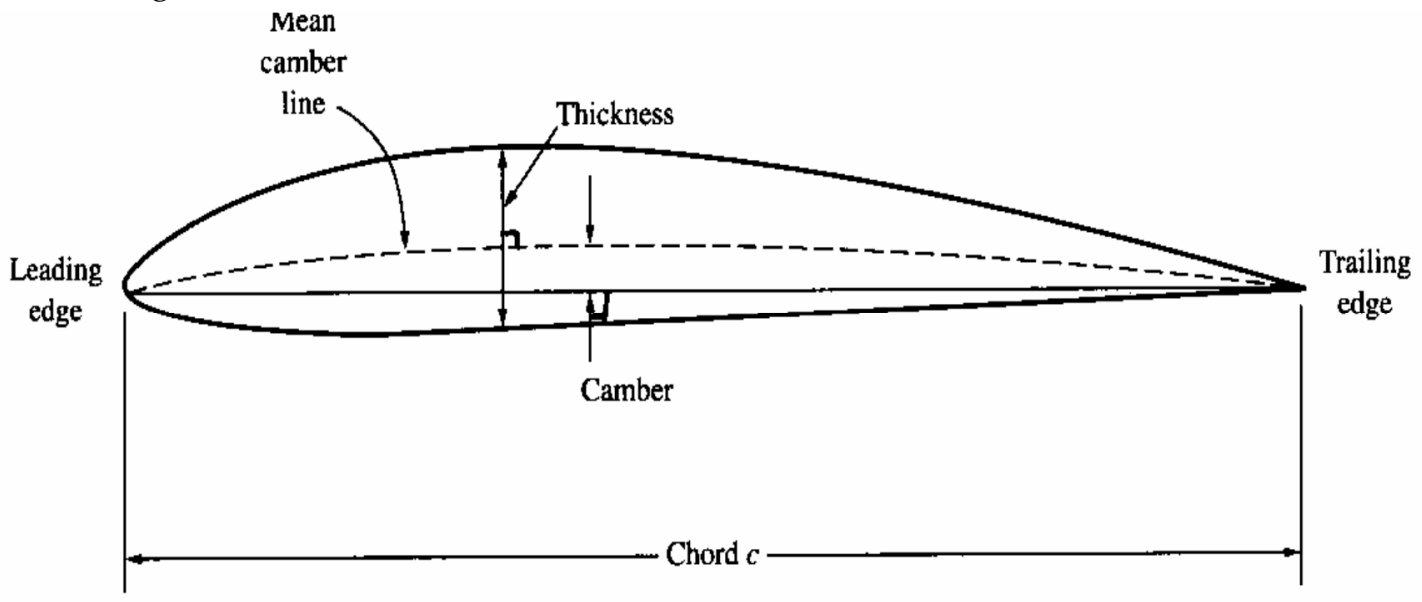

Fig. 2.1: A typical airfoil

M.C.L (mean camber line) is the path of points midway between the upper and lower surfaces as measured perpendicular to the M.C.L itself. The most forward point of the M.C.L is called Leading Edge. The most rearward point of the M.C.L is called Trailing Edge.The line which is straight connecting the leading edges and trailing edges is called the Chord line of the airfoil. The distance from the leading to the trailing edge measured along the chord line is simply called chord c.The camber is the highest distance between the M.C.L line and the chord line, measured with an angle of ninety degree to chord line.The distance between the upper and lower surfaces of the airfoil measured with an angle of ninety degree to the chord line is called thickness of the airfoil. Free stream velocity is the velocity of air far upstream of the airfoil. The direction of air is defined as relative wind. The angle between the relative wind and chord line is the angle of attack of the airfoil. The point at which moments of aerodynamic forces remains same for all angle of attack (moments do not vary with angle of attack). For NACA 4 digit series such as NACA 0012, NACA 2412 first digit indicates Maximum Camber in Hundredths of chord.Second digit indicates Location of maximum camber along the chord from the leading edge in tenths of chord.Last two digits indicates Maximum thickness of the airfoil in hundredths of chord

For NACA 4415 and chord of the airfoil $\mathrm{c}=1$

Maximum wing thickness $\mathrm{t}=$ last two digit $\times \% \mathrm{c}$

$$
\begin{aligned}
& =15 \times \frac{1}{100} \times 1 \\
& =0.15
\end{aligned}
$$

Distance from leading edge to maximum wing thickness $X_{1}=$ second digit $\times 10 \% c$

$$
\begin{aligned}
& =4 \times \frac{10}{100} \times 1 \\
& =0.4
\end{aligned}
$$


Maximum camber $\mathrm{f}=$ first digit $\times \% \mathrm{c}$

$$
=4 \times 0.01=0.04
$$

Maximum wing thickness

$Y_{t}=5 t\left(0.2969 \sqrt{x}-0.126 x-0.3516 x^{2}+0.2843 x^{3}-0.1015 x^{4}\right)$

From leading edge to maximum wing thickness

$$
\begin{gathered}
\frac{Y_{C}}{c}=\frac{f}{c}\left(\frac{1}{x_{1}^{2}}\right)\left[2 x_{1}\left(\frac{x}{c}\right)-\left(\frac{x}{c}\right)^{2}\right] \\
\text { Valid for } \quad 0 \leq \frac{x}{c} \leq x_{1}
\end{gathered}
$$

From maximum wing thickness to trailing edge

$$
\begin{aligned}
& \frac{Y_{C}}{c}=\frac{f}{c}\left(\frac{1}{\left(1-x_{1}\right)^{2}}\right)\left[\left(1-2 x_{1}\right)+2 x_{1}\left(\frac{x}{c}\right)-\left(\frac{x}{c}\right)^{2}\right] \\
& \qquad x_{1} \leq \frac{x}{c} \leq 1
\end{aligned}
$$

\section{Where}

$\mathrm{f}=$ maximum camber

$\mathrm{X}_{1}=$ distance from leading edge to highest wing thickness

$\mathrm{c}=$ chord of the airfoil

$\mathrm{t}=$ highest wing thickness

$\mathrm{yc}_{\mathrm{c}}=$ vertical distance between mean camber line and chord line.

For NACA 4415

From leading edge to highest thickness x1,

$$
\frac{Y_{C}}{c}=\frac{f}{c}\left(\frac{1}{x_{1}^{2}}\right)\left[2 x_{1}\left(\frac{x}{c}\right)-\left(\frac{x}{c}\right)^{2}\right]
$$$$
\frac{Y_{C}}{1}=\frac{.04}{1}\left(\frac{1}{.4^{2}}\right)\left[2 \times 0.4 \times\left(\frac{x}{1}\right)-\left(\frac{x}{1}\right)^{2}\right]
$$

$Y_{C}=0.2 x-0.25 x^{2}$

From highest wing thickness to trailing edge,

$$
\frac{Y_{C}}{c}=\frac{f}{c}\left(\frac{1}{\left(1-x_{1}\right)^{2}}\right)\left[\left(1-2 x_{1}\right)+2 x_{1}\left(\frac{x}{c}\right)-\left(\frac{x}{c}\right)^{2}\right]
$$

$$
\frac{Y_{C}}{1}=\frac{.04}{1}\left(\frac{1}{(1-0.4)^{2}}\right)\left[(1-2 \times 0.4)+2 \times 0.4\left(\frac{x}{1}\right)-\left(\frac{x}{1}\right)^{2}\right]
$$

$$
Y_{C}=0.222+0.8888 x-0.111 x^{2}
$$


For maximum wing thickness

$$
\begin{aligned}
& Y_{t}=5 t\left(0.2969 \sqrt{x}-0.126 x-0.3516 x^{2}+0.2843 x^{3}-0.1015 x^{4}\right) \\
& =5 \times 0.15\left(0.2969 \sqrt{x}-0.126 x-0.3516 x^{2}+0.2843 x^{3}-0.1015 x^{4}\right) \\
& \left.=0.222 \sqrt{x}-0.0945 x-0.2637 x^{2}+0.213 x^{3}-0.076 x^{4}\right)
\end{aligned}
$$

Programming language is applied for designing the regular surface profile of the NACA 4415. The thickness and chord length of the model are $3.14 \mathrm{~cm}$ and $21 \mathrm{~cm}$ respectively. The span length was chosen to be $21 \mathrm{~cm}$ a considerably large value. For ensuring the aerodynamic characteristics of an airfoil the trailing edge of the model have a sharp edge form

\section{Program for Data Generation:}

The data for obtaining the airfoil profile is generated by programming using $\mathrm{C}$ compiler. The following code is used to generate data for designing NACA-4415 profile using solid works software. \#include<stdio.h> \#include<conio.h> \#include<math.h> void main() \{

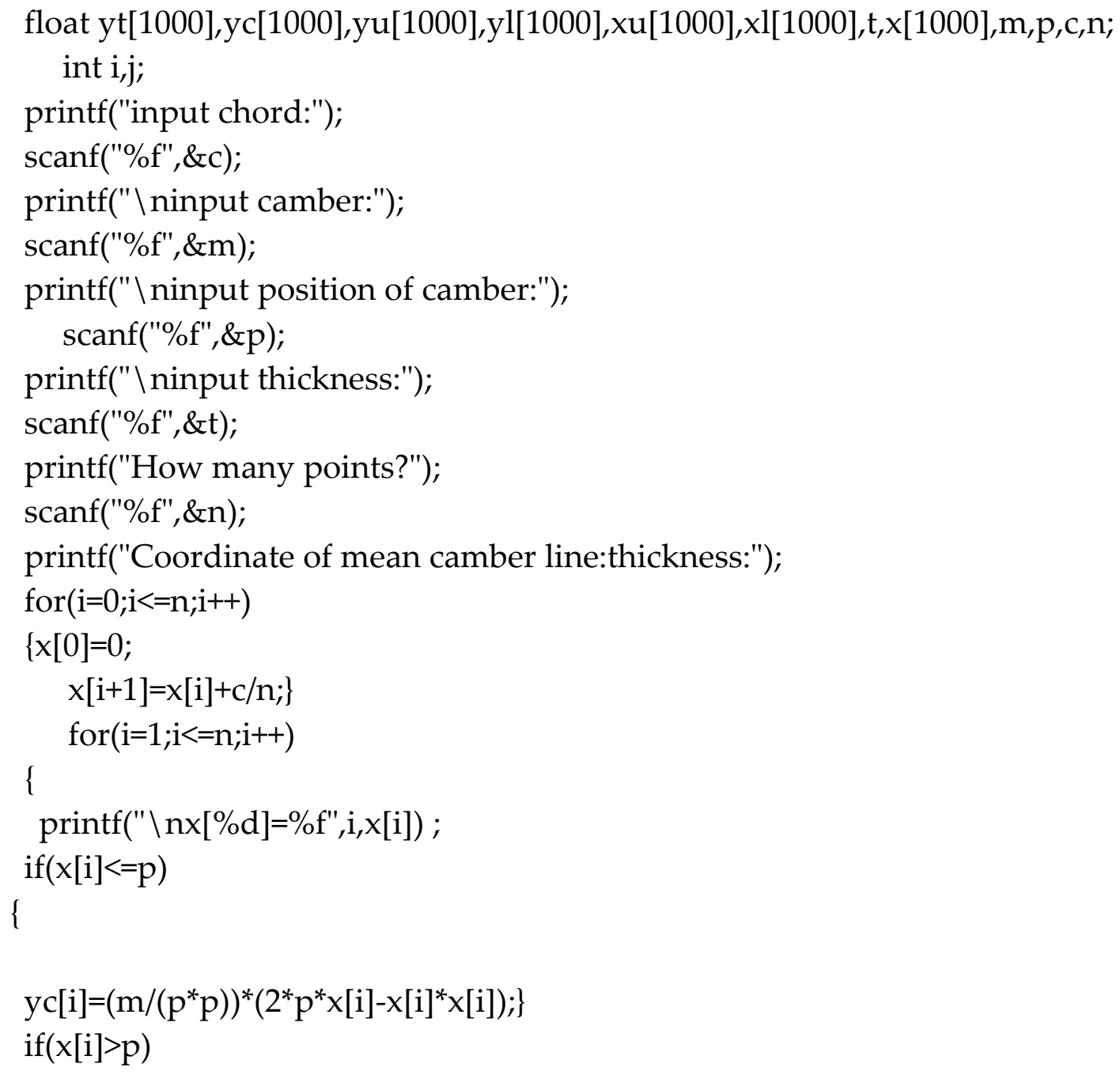




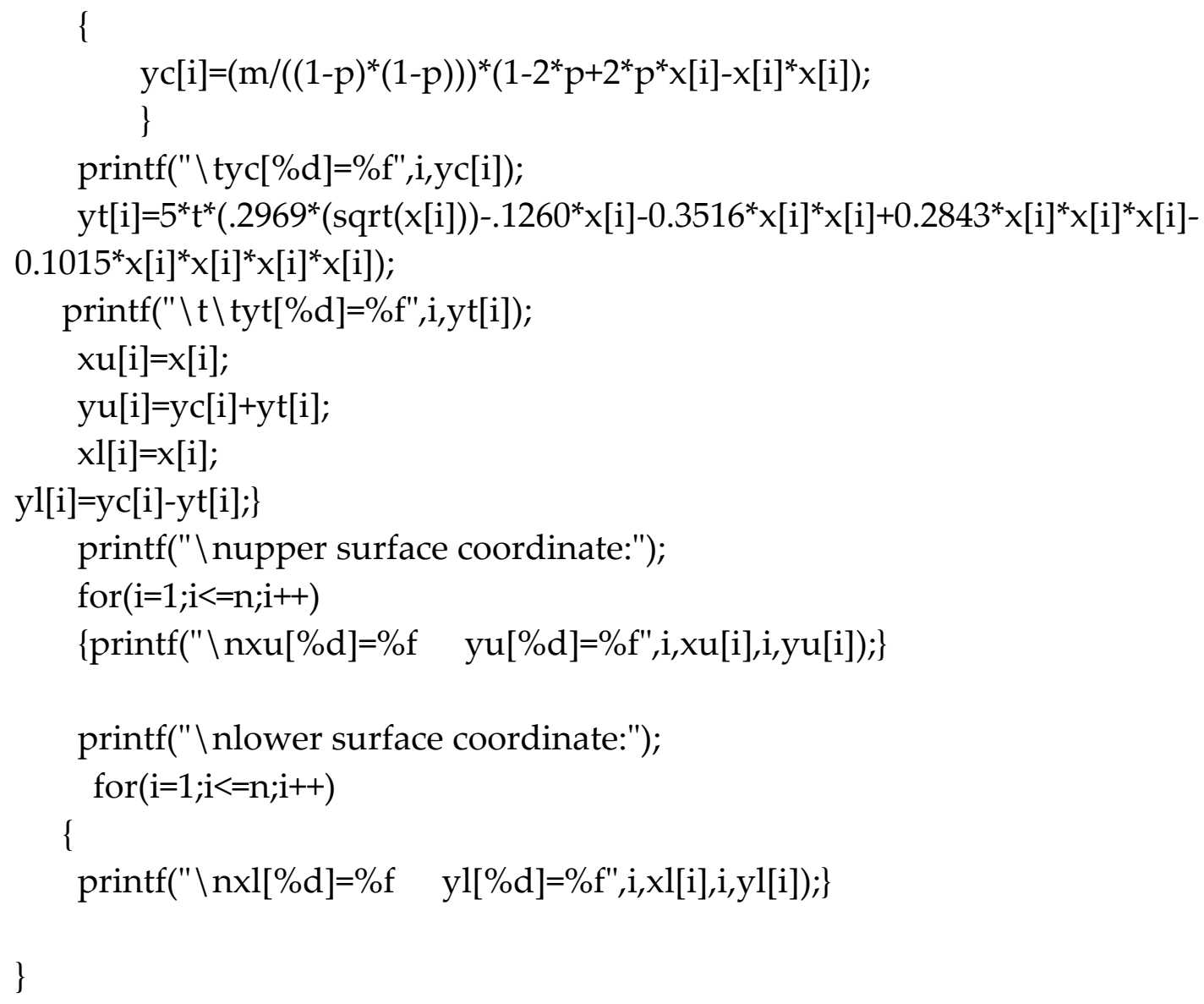

\section{Model Construction:}

The design was made by using solid works software. Five different models with spherical outward, spherical inward, hexagonal, Circular and with Combination of circular and hexagonal dimples have been constructed.Wood was used for model preparation. As wood is economical to use so it will be convenient for the construction of dimpled airfoil models. The chord of regular surface airfoils is $21 \mathrm{~cm}$ and the chord of dimpled surface airfoil is also $21 \mathrm{~cm}$.

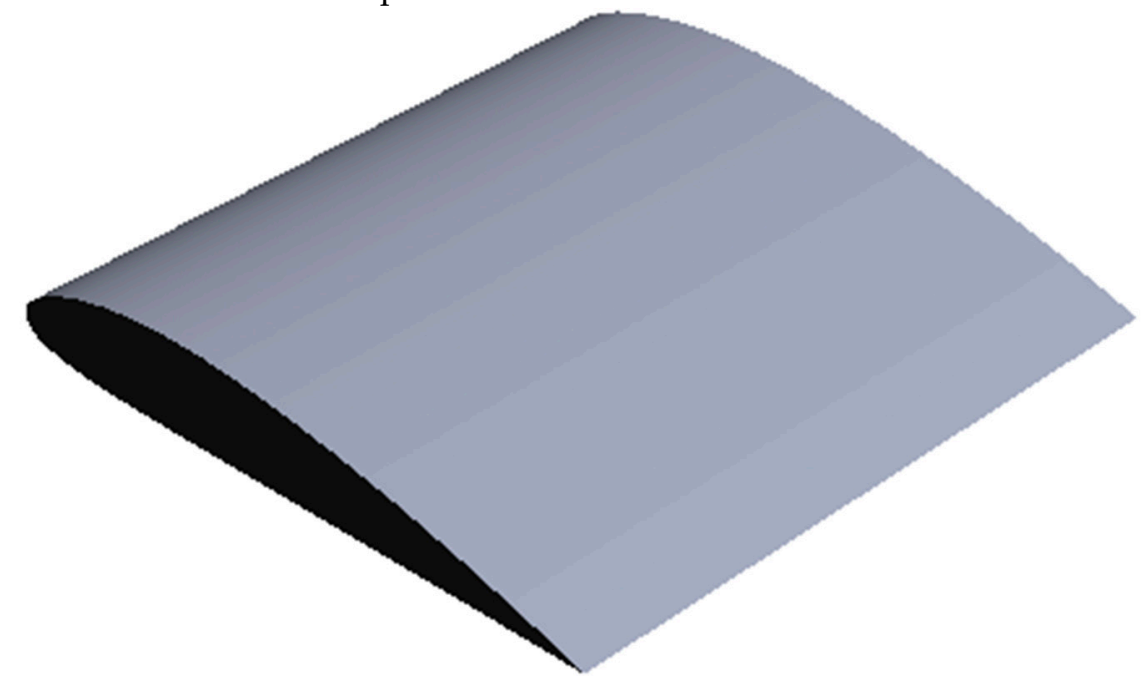

Fig 4.1: Three dimensional view of NACA 4415 airfoil obtained from solid works. 


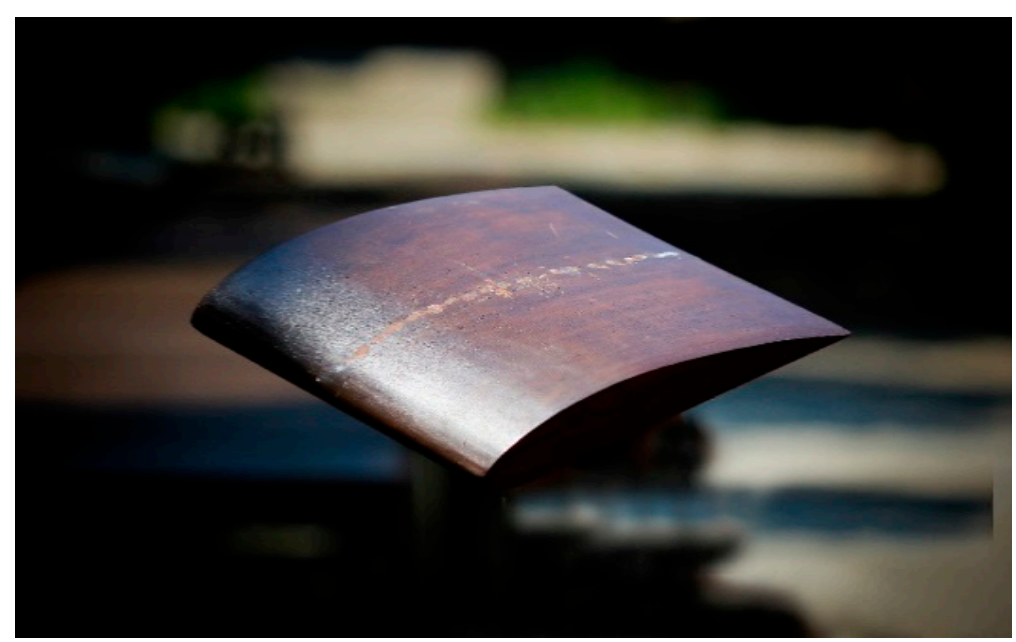

Fig.4.2.Prepared model without modifications.

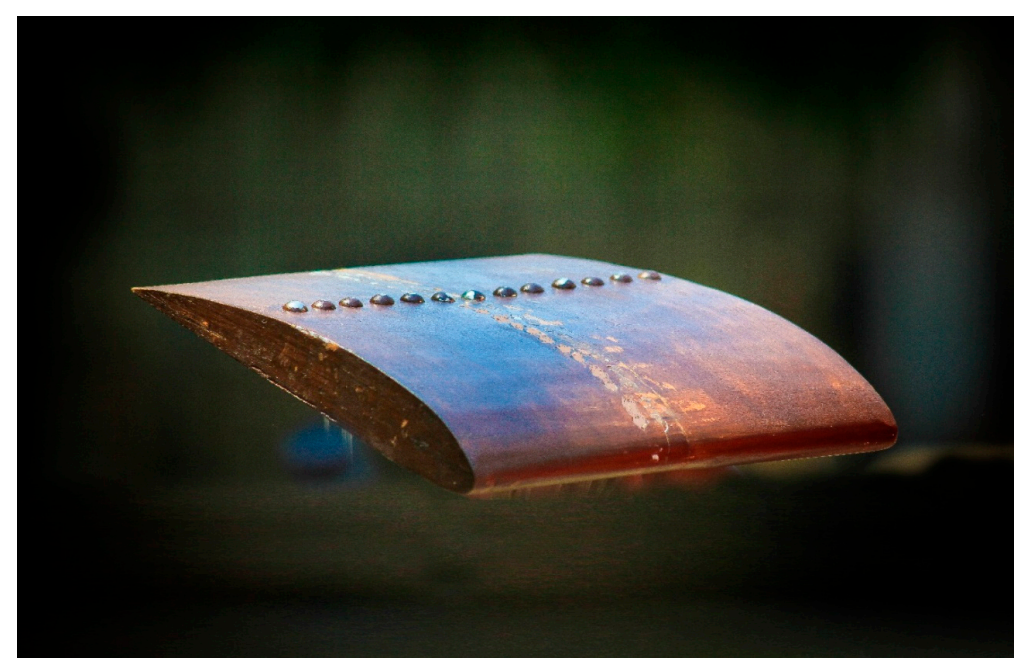

Fig.4.3. Prepared model with outward spherical dimples.

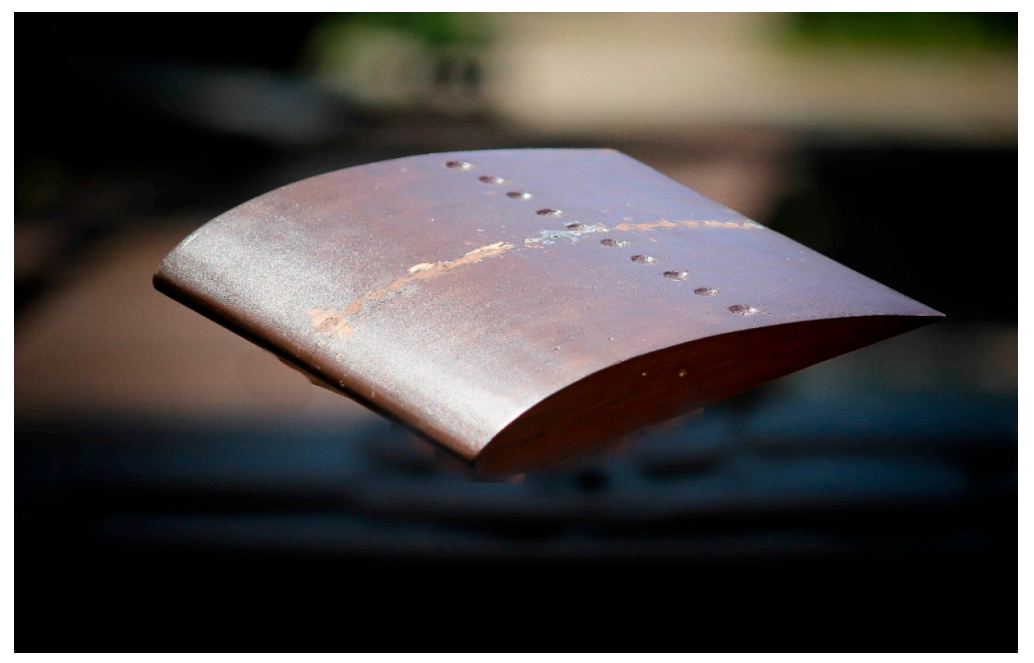

Fig.4.4. Prepared model with inward spherical dimples 


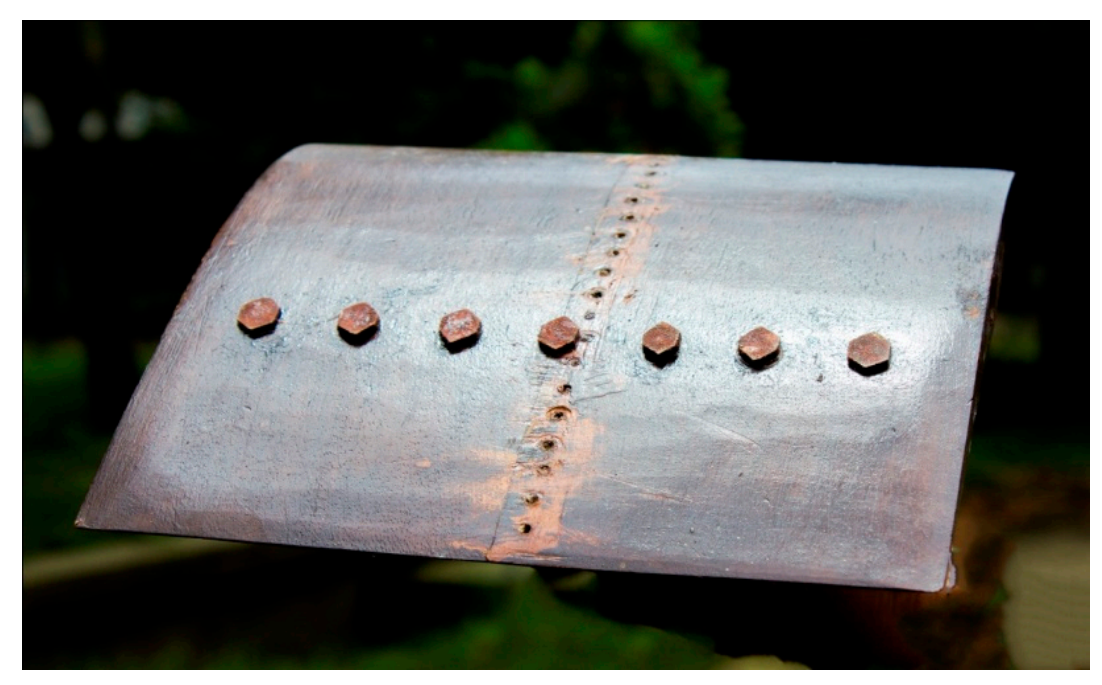

Fig.4.5. Prepared model with hexagonal dimples

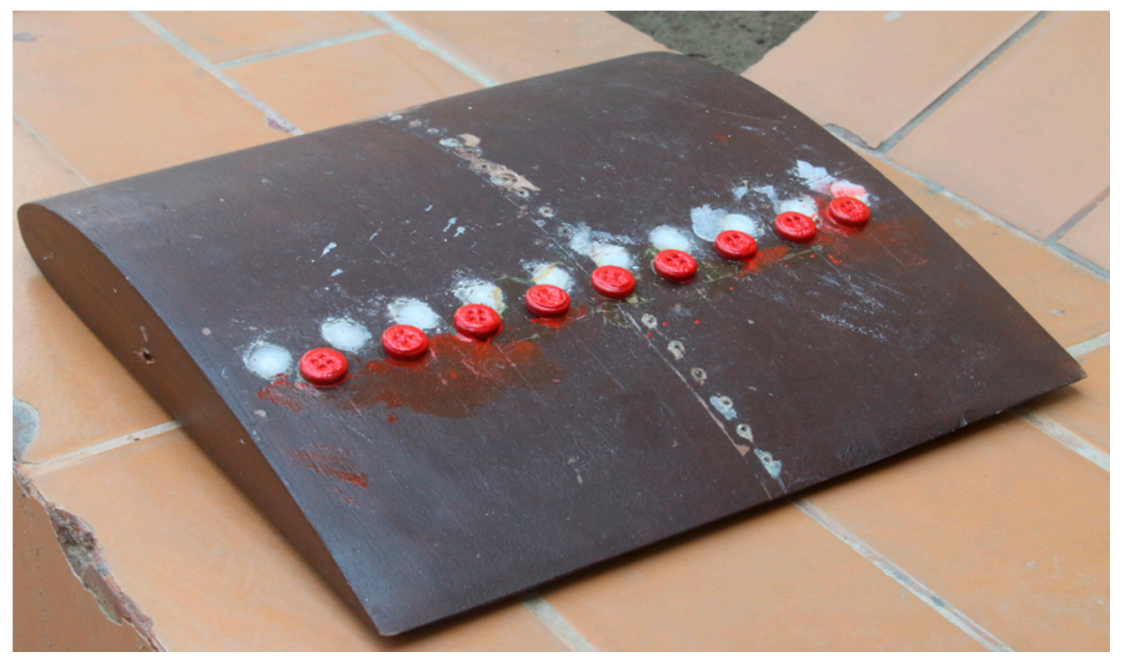

Fig.4.6. Prepared model with circular dimples

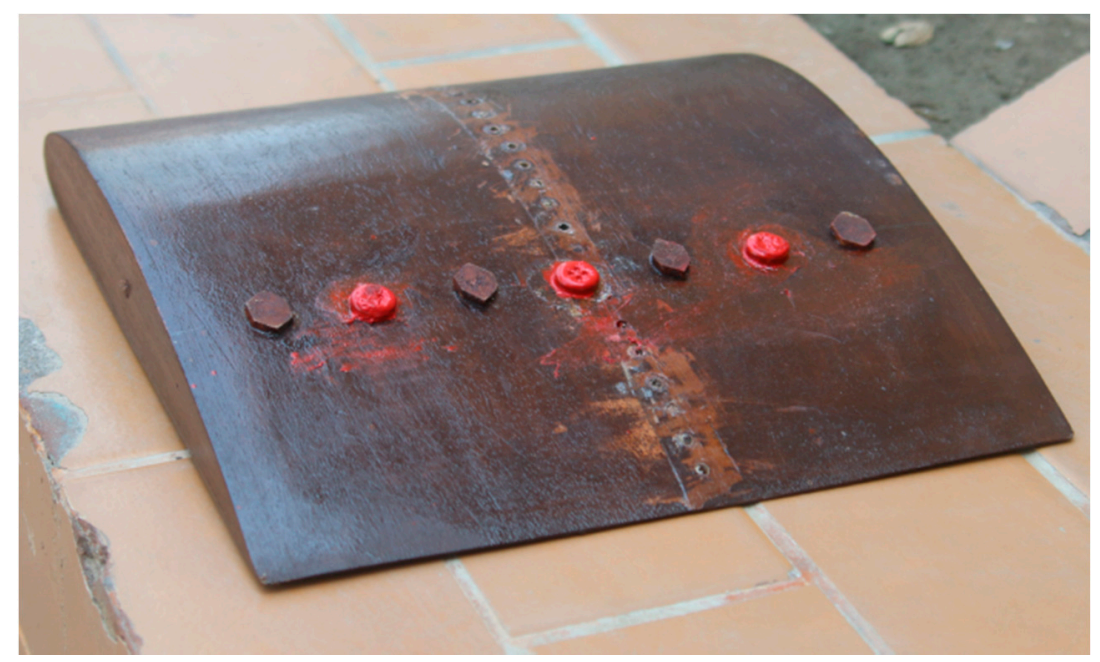

Fig.4.7. Prepared model with combination of hexagonal and circular dimples

\section{Conclusions}


Five different models with spherical outward, spherical inward, hexagonal, Circular and with Combination of circular and hexagonal dimples have been constructed .Delayed flow separation can be observed if the dimpled airfoils are tested. At a higher angle of attack attached flow on the dimpled surface will be seen. The lift of dimpled surface airfoil will also better than the regular airfoils. Further investigations using these models are suggested.

\section{References}

[1] John D. Anderson, J.R,"Introduction to Flight", Third Edition, Mc Graw-Hill International Editions, Aerospace Science Series

[2] Dr. P. N. Modi \& Dr. S. M Seth, "Hydraulics and Fluid Mechanics Including Machine" (In SI Unit), new edition 2005-2006, Standard Book House

[3] William H. Rae, Jr. \& Alan pope, "Low Speed Wind Tunnel Testing", Second Edition, A Wiley Inter science Publication

[4] Charles E. Dole,"Flight Theory and Aerodynamics",A Wiley Interscience Publication

[5] Bernard Etkin,"Dynamics of Flight- Stability and Control", Third edition, John Wiley \& Sons, INC

[6] Dr. M.Mashud, "Role of partially bumpy surface to control the flow separation of an airfoil", ARPN Journal of Engineering and Applied Sciences, Vol. 7, No. 5, May 2012.

[7] Davies, J.M.,"The aerodynamics of golf balls", J of Applied Physics, 1949, 20, (9), PP 821-828

[8] Nickerson, J.D., "A study of vortex generators at low Reynolds numbers”, AIAA, 1986, AIAA86-0155

[9] Md.Amzad Hossain,Md. Nizam Uddin,Rubiat Mustak,“Experimental Study of Aerodynamic Characteristics Of Airfoils Using Different Shaped Dimples", The International Journal Of Engineering And Science (IJES) ,Volume 4, Issue 1, Pages PP.13-17,2015

[10] Rubiat Mustak, Md. Harun-Or-Rashid Molla, Mohammad Mashud, "Improvement of Aerodynamic Characteristics of an Airfoil by Surface Modification",American Journal of Engineering Research (AJER),Volume 6,Issue 3,PP.07-14,2017.

[11] Rubiat Mustak, Md.Habib Ullah Khan, Md.Mahbubur Rahman, Mohammad Mashud," Investigation of Slipstreaming Effect between a Semi trailer-truck and a Sedan Car ", International Journal of Scientific \& Engineering Research(IJSER), Volume 8, Issue 2,Pages PP.676-678,February-2017 